\title{
Tiaojing Cuyun Recipe Enhances Pregnancy Outcome via the VEGF/PI3K/AKT/eNOS Signaling Pathway in EID Mice
}

\author{
Hongli Huang $\mathbb{D},{ }^{1}$ Lei Xia $\mathbb{D}^{1},{ }^{1}$ Yanqiu Xia $\mathbb{D}^{1},{ }^{1}$ Yunping Yan $\mathbb{D}^{1},{ }^{1}$ Zhuojun Jiang $\mathbb{D}^{1}$ \\ Pei Zhao ${ }^{2}{ }^{2}$ and Li Dong ${ }^{1}$ \\ ${ }^{1}$ Yueyang Hospital of Integrative Traditional Chinese and Western Medicine, \\ Shanghai University of Traditional Chinese Medicine, China \\ ${ }^{2}$ The Public Experiment Platform, Shanghai University of Traditional Chinese Medicine, China
}

Correspondence should be addressed to Pei Zhao; zp1737@sina.com and Li Dong; yydongli@163.com

Received 2 August 2021; Accepted 9 February 2022; Published 24 February 2022

Academic Editor: Azizah Ugusman

Copyright ( $) 2022$ Hongli Huang et al. This is an open access article distributed under the Creative Commons Attribution License, which permits unrestricted use, distribution, and reproduction in any medium, provided the original work is properly cited.

\begin{abstract}
Purpose. In this study, we evaluated the effect of Tiaojing Cuyun Recipe (TJCYR) on embryo implantation dysfunction- (EID-) induced damage of endometrial receptivity in mice and investigated the mechanisms underlying the effect. Methods. The main compounds of TJCYR were identified by high-performance liquid chromatography (HPLC). One hundred and twenty pregnant mice were randomly divided into six groups: control, EID only, progesterone (Prog)+EID, TJCYR-low-dose+EID, TJCYR-medium-dose+EID, and TJCYR-high-dose+EID. Mifepristone was injected to make the EID model. On the fourth day of pregnancy, serum was obtained to analyze hormone level by radioimmunoassay, the uterus was collected to analyze morphology by hematoxylin and eosin (H\&E) and scanning electron microscopy (SEM), and a combination of immunofluorescence and Western blot was used to identify the related proteins. On the eighth day of pregnancy, the mice were sacrificed and the number of uterus-implanted blastocysts was counted. Results. Treatment with TJCYR significantly improved the number of implanted sites, the number of well-developed pinopodes, and microvascular formation in the mice. Moreover, TJCYR significantly activated PI3K/Akt/eNOS signaling pathways to promote angiogenesis, resulting in significantly improved endometrial receptivity and fertility outcomes when compared to the model group. Conclusion. These findings demonstrate that TJCYR was able to protect embryo implantation of EID mice due to TJCYR-mediated improvement in endometrial receptivity by promoting endometrial angiogenesis.
\end{abstract}

\section{Introduction}

The incidence of infertility has been a global health problem among women [1]. While access to assisted reproductive technology (ART) has overcome the majority of infertility causes, success rates have stagnated at around 30\% [2]. Successful pregnancy inevitably depends on high embryo quality and good endometrial receptivity. During pregnancy, the endometrium apical surface undergoes several morphological, molecular, and biochemical changes to provide a favorable environment for embryo implantation, thereby creating an effective maternal-fetal interaction [3]. Thus, endometrial receptivity is key to raising the pregnancy rate in women with infertility [4-6]. Attempts at focusing on improvement of endometrial receptivity have been made in recent years, such as enhancing microcirculation and trophoblast invasion [7]. During the reproductive processes, the supply of essential nutrients and oxygen from vasculature is very important for the development of maternal-fetal interaction. It has been reported that the endometrium thickens with mature vascular network and increased blood flow, which reflect sufficient endometrial receptivity and, in particular, determine the endometrial response to the blastocyst at the early stage of embryo implantation [8]. Thus, enhanced endometrial angiogenesis is one of the important biological events during the reproductive cycle and pregnancy.

Traditional Chinese Medicine has gained wide acceptance due to its advantages of multifactorial and multitarget actions. In our clinical practice, we found that Tiaojing Cuyun Recipe (TJCYR) clearly enhanced the rate of 
pregnancy in women with infertility. Some studies reported that TJCYR may regulate follicular development and elevate the pregnancy rate in anovulatory infertility patients with kidney deficiency syndrome and significantly improve the ovulation rate and the reproductive function of the endocrine axis in androgen-sterilized rats $[9,10]$. It has been reported that Bushen Huoxue Formula, which is composed of TJCYR, can improve the ACT-INH-FS system in patients with polycystic ovary syndrome with kidney deficiency and blood stasis [11]. Experimental studies have also demonstrated that Epimedium brevicornu Maxim. and Morinda officinalis How. improve endometrial receptivity in ovulation stimulation (OS) in EID mice through significant improvements in the spatial and temporal expression of pinopodes, accompanied by a significantly increased number of embryonic implantation sites [12]. These effects are desirable and satisfactory, but the therapeutic mechanism of TJCYR is not yet fully elucidated. The aim of this study was to provide a detailed account of the beneficial effect of TJCYR on EID-induced damage to endometrial receptivity in mice and to investigate its mechanism of action. From this perspective, we attempted to find novel candidates from traditional medicinal herbs to enhance the pregnancy outcome.

\section{Materials and Methods}

2.1. Medicine Preparation. TJCYR consists of seven herbs (Table 1). All herbs were supplied by the Shanghai Kangqiao Chinese Medicine Tablet Co., Ltd. (Shanghai, China). The decoction component mixture was added with water of 4 times volume, boiled twice, concentrated using a rotary evaporator, and attained the equivalent crude content of $2.0 \mathrm{~g} / \mathrm{mL}$. The concentrations of $12 \mathrm{~g} / \mathrm{kg}, 24 \mathrm{~g} / \mathrm{kg}$, and $48 \mathrm{~g} /$ $\mathrm{kg}$ of the preparations were designated as TJCYR-L, TJCYR-M, and TJCYR-H, respectively.

\subsection{Identification of Chemical Constituents in TJCYR by} High-Performance Liquid Chromatography (HPLC). The active ingredients of TJCYR were examined using the 1200 series HPLC device (Agilent Technologies, Santa Clara, CA, USA) with an autosampler (G1329B), thermostatted column compartment (G1316A), quaternary pump (G1311A), photodiode array detector (G1315D), and degasser (G1322A). HPLC was performed on an Apollo C18 $(4.6 \times 250 \mathrm{~mm}$; particle size, $5 \mu \mathrm{m}$; GRACE, Columbia, Maryland, USA) with a mobile phase of acetonitrile (A) $-0.1 \%(v / v)$ and phosphoric acid (B) for gradient elution (0-70 min, $1-40 \% \mathrm{~A} ; 70-90 \mathrm{~min}, 40-80 \% \mathrm{~A})$. The detection wavelength was $260 \mathrm{~nm}$, and the flow rate was $1 \mathrm{~mL} / \mathrm{min}$. The column temperature was $30^{\circ} \mathrm{C}$, and the injection volume was $10 \mu \mathrm{L}$. The standard solutions of Calycosin-7-glucoside, Acteoside, Salvianolic acid B, Icariin, Tanshinone IIA, and sample were filtered with a $0.45 \mu \mathrm{m}$ membrane filter before subjecting them to HPLC analysis.

2.3. Reagents. Mifepristone and progesterone were obtained from the pharmacy department of Yueyang Hospital of Integrative Traditional Chinese and Western Medicine; hematoxylin-eosin, Antifade Polyvinylpyrrolidone Mount- ing Medium, and nitric oxide (NO) assay kit were purchased from Beyotime (China); the antibodies $\operatorname{ER} \alpha, \operatorname{ER} \beta 1$, $\mathrm{PR}$, Integrin $\alpha \mathrm{V}$, Integrin $\beta 3$, OPN, LIF, eNOS, and p-eNOS were purchased from Abcam (UK); vascular endothelial growth factor (VEGF) and glyceraldehyde-3phosphate dehydrogenase (GAPDH) were purchased from Proteintech (USA); Alexa Fluor $488 \mathrm{~nm}$, Alexa Fluor $555 \mathrm{~nm}$, Akt, p-Akt, and $\beta$-tubulin were purchased from CST (USA); Lycopersion Esculentum Lectin was purchased from Vector Laboratories (USA); DAPI was purchased from Sigma (USA); chemiluminescence detection kit was purchased from Millipore (USA).

2.4. Animals. Adult female and male Kunming mice (weighing 25-28 g) were purchased from Beijing Vital River Laboratory Animal Technology Co, Ltd. (Beijing, China; SCXK(J)2016-0011). All experimental protocols were approved by the Animal Care and Use Committee of Shanghai University of Traditional Chinese Medicine (Shanghai, China, PZSHUTCM200703008). The mice were housed in cages separately with controlled temperature and humidity, $12 \mathrm{~h}$ light-dark periods, and free access to water and a standard diet. The two estrous cycles were observed by using vaginal smears in female mice before the treatment.

2.5. Treatments and Establishment of EID. The female mice were mixed with male mice in a ratio of $2: 1$ to mate overnight in the independent cages. The following morning, the presence of a vaginal plug was considered as an indicator of successful copulation, and this day was classified as day 1 of pregnancy (Pd1). The pregnant mice were then randomly divided into six experimental groups ( $n=20$ in each group), including the control, EID only, progesterone (Prog)+EID, TJCYR-low-dose+EID, TJCYR-medium-dose+EID, and TJCYR-high-dose+EID. In the control and EID-only groups, the mice were given intragastric administration of physiological saline solution once daily for 4 days; the treatment group was given Prog and TJCYR, respectively. Mifepristone ( $0.1 \mathrm{mg} /$ mouse) was subcutaneously injected at $\mathrm{Pd} 4$ in the morning, to establish the EID model [13]. Mice were sacrificed on Pd4 and Pd8, respectively. We obtained blood samples from the orbital vein on $\mathrm{Pd} 4$, and the serum was collected to detect hormone levels. The number of implantation sites on Pd8 was recorded. The uteri were harvested on $\mathrm{Pd} 4$ to evaluate endometrial receptivity and the potential mechanism. The procedure followed in this experiment is shown in Figure 1(a).

2.6. Hematoxylin and Eosin (HळE) Staining. The uteri were dissected and fixed in $4 \%$ paraformaldehyde and embedded in paraffin, then serially sectioned at a thickness of $5 \mu \mathrm{m}$. Sections were stained with H\&E for morphological measurements. The slides were mounted after H\&E staining and were examined using an optical microscope (Stemi DV4, Carl Zeiss, Oberkochen, Germany).

2.7. Scanning Electron Microscopy (SEM). The endometria were sliced and fixed in $1.25 \%(w / v)$ glutaraldehyde solution and $1 \%$ osmium tetroxide at $4^{\circ} \mathrm{C}$ for $2 \mathrm{~h}$, respectively. The samples were dehydrated through graded concentrations of 
Table 1: Tiao Jing Cu Yun Recipe (TJCYR) components.

\begin{tabular}{lcrrr}
\hline Chinese term & Generic name & Scientific name & Weight (g) & Product lot \\
\hline Dangshen & Codonopsis pilosula (Franch.) Nannf. & Codonopsis radix & 20 & 171011 \\
Danshen & Salvia miltiorrhiza Bge. & Salviae miltiorrhizae radix et rhizoma & 20 & 170701 \\
Danggui & Angelica sinensis (Oliv.) Diels & Angelicae sinensis radix & 20 & 170606 \\
Huangqi & Astragalus membranaceus (Fisch.) Bunge. & Astragali radix & 20 & 160829 \\
Shudihuang & Rehmannia glutinosa (Gaert.) Libosch.ex Fisch.et Mey. & Rehmanniae radix praeparata & 15 & 170420 \\
Bajitian & Morinda officinalis How. & Morindae officinalis radix & 12 & 171219 \\
Yinyanghuo & Epimedium brevicornu Maxim. & Epimedii folium & 12 & 170723 \\
\hline
\end{tabular}

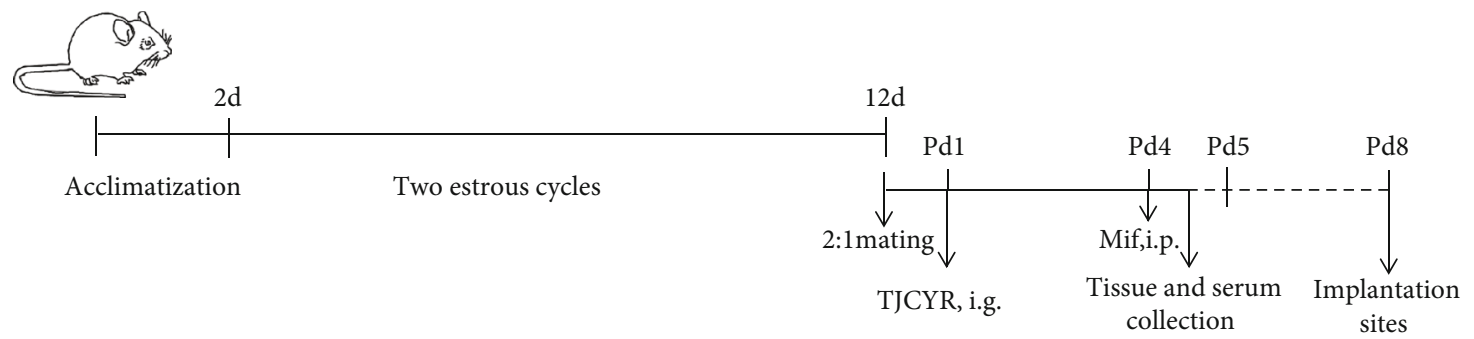

(a)

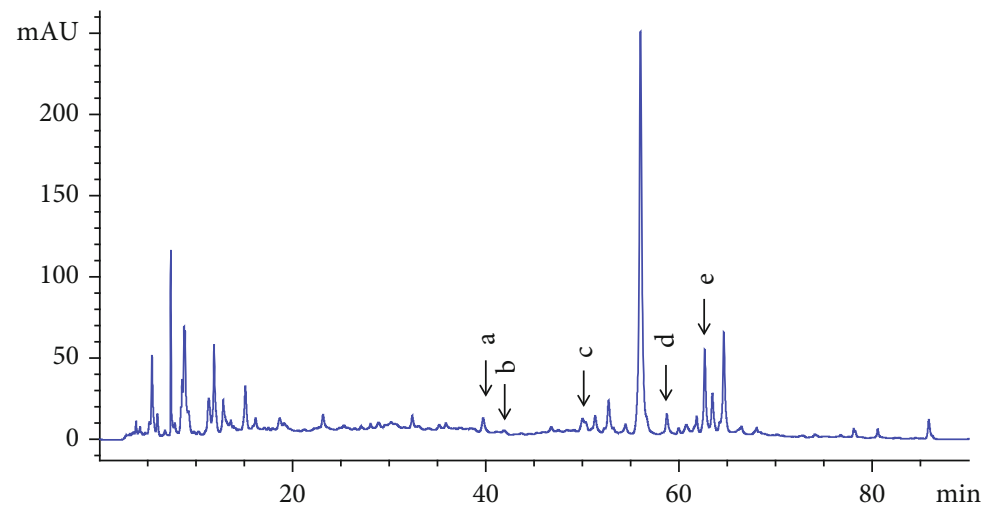

(b)

FIgure 1: Study scheme and the fingerprinting of TJCYR. (a) Schematic diagram showing the experimental protocol; (b) characteristic fingerprint of TJCYR as analyzed by HPLC. Pd: day of pregnancy; EID: embryo implantation dysfunction; TJCYR: Tiaojing Cuyun Recipe; HPLC: high-performance liquid chromatography; a: Calycosin-7-glucoside; b: Acteoside; c: Salvianolic acid B; d: Icariin; e: Tanshinone IIA.

ethanol and subsequently dried in a critical-point drier with carbon dioxide, then mounted onto the specimen holder and coated with gold palladium. Finally, all specimens were observed under the SEM (SU8010, Hitachi Instruments, Tokyo, Japan).

2.8. Immunofluorescence Staining. Isolated uteri were frozen in optimal cutting temperature compound (OCT) in liquid nitrogen and were serially frozen-sectioned at $8 \mu \mathrm{m}$ thick. Nonspecific proteins were blocked with 5\% fetal bovine serum for $1 \mathrm{~h}$ at room temperature (RT). The sections were then incubated with primary antibodies (ER $\alpha, 1: 200$; $\mathrm{ER} \beta 1,1: 500$; PR, $1: 200$; Integrin $\alpha \mathrm{V}, 1: 200$; Integrin $\beta 3$, $1: 100$; OPN, 1:500; LIF, 1:500) or fluorescence probe (Lycopersion Esculentum Lectin) overnight at $4^{\circ} \mathrm{C}$. The next day, the sections were incubated with the Alexa Fluor
$488 \mathrm{~nm}$ (green) or Alexa Fluor $555 \mathrm{~nm}$ (red) for $1 \mathrm{~h}$ at RT in dark, then stained with DAPI (blue). After rinsing three times with PBST, the slides were mounted with Antifade Polyvinylpyrrolidone Mounting Medium and then covered with coverslips. Finally, images were obtained using a Carl Zeiss LSM800 confocal microscope (Carl Zeiss Microscope GmbH, Jena, Germany).

2.9. Quantification of NO Content. NO concentration in uterus tissue was measured with the NO assay kit following the user manual. The quantitative determination of nitrite levels represents NO content.

2.10. Western Blotting. The uterus tissue was mixed with lysis buffer and the protease inhibitor, then the samples were homogenized and centrifuged, and the supernatants were 
collected. After determining the protein concentration, the lysates were separated by $10 \%$ SDS-PAGE and then transferred to PVDF $(0.45 \mu \mathrm{m}$, EMD Millipore, Billerica, MA, USA). The membranes were blocked with $5 \%$ nonfat milk for $1 \mathrm{~h}$ at RT and then probed overnight at $4^{\circ} \mathrm{C}$ with primary antibodies $\operatorname{ER} \alpha(1: 1000), \operatorname{ER} \beta 1(1: 1000)$, PR $(1: 1000)$, Integrin $\alpha \mathrm{V}(1: 1000)$, Integrin $\beta 3(1: 1000)$, OPN $(1: 500)$, LIF $(1: 1000)$, VEGF $(1: 1000)$, Akt $(1: 1000)$, p-Akt $(1: 1000)$, eNOS $(1: 1000)$, p-eNOS $(1: 1000)$, GAPDH $(1: 3000)$, or $\beta$-tubulin $(1: 1000)$. Next, the membranes were washed and incubated with the appropriate secondary antibody for $1 \mathrm{~h}$ at RT. Finally, signals were detected using a chemiluminescence detection kit (EMD Millipore, Billerica, MA, USA) and the FluorChem E imaging system (ProteinSimple, San Francisco, CA, USA); the protein band densities were quantified by using an image analysis system (Alpha View SA, ProteinSimple, San Francisco, CA, USA) and expressed as ratios to GAPDH or $\beta$-tubulin.

2.11. Statistical Analysis. All results were expressed as means \pm SEM. Differences were compared by one-way analysis of variance (ANOVA) and were considered significant at $P<0.05$. All statistical tests were performed with GraphPad Prism software version 5.0.

\section{Results}

3.1. Quality Evaluation of TJCYR by HPLC. According to the Pharmacopoeia of the People's Republic of China (2020 version), the indicative quality control components of the TJCYR are Calycosin-7-glucoside (standard substance for Astragalus membranaceus (Fisch.) Bunge), Acteoside (standard substance for Rehmannia glutinosa (Gaert.) Libosch.ex Fisch.et Mey.), Salvianolic acid B (standard substance for Salvia miltiorrhiza Bge.), Icariin (standard substance for Epimedium brevicornu Maxim.), and Tanshinone IIA (standard substance for Salvia miltiorrhiza Bge.), respectively. In this study, we established a method for determination of multiple indicator compounds by HPLC, which can effectively determine multiple indicator components in the compound at the same time. The results are shown in Figure 1(b). HPLC chromatographic results showed that the indicator components of these TCM components in the recipe were effectively transferred to the extract during water decocting. That is, TJCYR could play efficacy in the mice treated with TJCYR by intragastric administration. It well guaranteed the quality and pharmacodynamics of TJCYR.

3.2. TJCYR Enhancement of Blastocyst Implantation. The numbers of blastocyst sites were recorded on Pd8. As indicated in Figure 2, the embryos appear well-developed and show a string-of-beads arrangement in the control group, while the embryos were markedly tiny and runtish in the EID group, which suggested that the EID was a successful model. Treatment with TJCYR increased the implantation numbers and profoundly promoted the development and distribution of the embryos, especially in mice administered with TJCYR-H, so this treatment was used in the following study.
3.3. TJCYR Improved EID-Induced Endometrium Morphological Changes. For HE and SEM, all samples were assigned a random number to minimize technical and investigator bias by a double-blinded way. The HE and SEM were read by the professional of pathology and electron microscope in Science and Technology Experiment Center of Shanghai University of Traditional Chinese Medicine, respectively. And then, data was analyzed to reveal the result of morphological changes in every group. As shown in Figure 3(a), H\&E staining was used to evaluate the pathological changes of the endometrium. In the EID-only group, loose endometrial stromal tissue and insufficient glands and vessels were found, in contrast to the mice treated with TJCYR. To further assess the morphological TJCYR-related changes on EID, we investigated the effect of TJCYR on the pinopodes using SEM. As shown in Figure 3(b), in the control group, the majority of well-developed pinopodes were evenly distributed over the endometrial epithelial surfaces, while on EID-induced surfaces, well-developed pinopodes were sparse and a few developing pinopodes were seen. However, the pinopodes in this group improved significantly following treatment with TJCYR. The results suggest that TJCYR can improve the endometrial morphology and vasculature in an EID model, with benefits for embryo implantation.

3.4. The Effect of TJCYR on Hormones and Hormone Receptors. The receptive mouse uterus displays stromal proliferation and epithelial differentiation, indicating readiness for blastocyst implantation, and is governed by ovarian hormones, such as $17 \beta$-estradiol $\left(\mathrm{E}_{2}\right)$ and progesterone $\left(\mathrm{P}_{4}\right)$. We tested the serum content of $\mathrm{E}_{2}$ and $\mathrm{P}_{4}$ in the serum. For the hormonal analysis, all samples were also randomly assigned; the test of hormone was conducted by Shanghai Xinfan Biological Technology Co., Ltd. And the results were analyzed in a double-blind way. We found similar $\mathrm{E}_{2}$ level in the EID only and control groups, but the level of $\mathrm{P}_{4}$ was notably reduced in the EID only group. Treatment with TJCYR may inhibit the $\mathrm{P}_{4}$ decrease induced by EID. The primary mediators of these $\mathrm{E}_{2}$ - and $\mathrm{P}_{4}$-induced events are their receptors, estrogen receptor (ER) and progesterone receptor (PR). We also test the expression of ER and PR by immunofluorescence (IF) and Western blot (WB). ER include ER-alpha $(\mathrm{ER} \alpha)$ and ER-betal (ER $\beta 1)$. The result of IF showed that the fluorescence intensity of ER $\alpha$ in the EID-only group was slightly weaker than in the controls, and no difference was found in the fluorescence intensity of ER $\beta 1$ between the EID-only group and the control group. However, the fluorescence intensity of PR was clearly weaker in the EIDonly group than that in the control group, and treatment with TJCYR could enhance the expression of PR. In the $\mathrm{WB}$, we also found that PR protein was clearly decreased in the EID-only group and that TJCYR could reverse that change. These results show that the changes in $\mathrm{P}_{4}$ and $\mathrm{PR}$ were clearly apparent in the EID model and that treatment with TJCYR could regulate $\mathrm{P}_{4}$ and PR (Figure 4).

3.5. The Treatment of TJCYR Increases Endometrial Receptivity-Related Markers. Several molecular markers are 


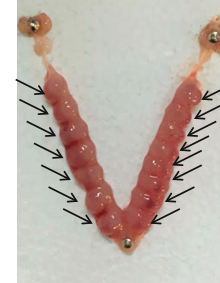

Con

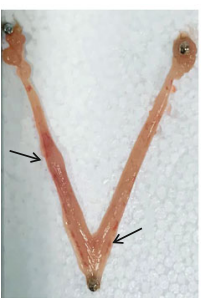

TJCYR-L+EDI

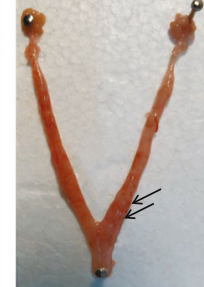

EDI

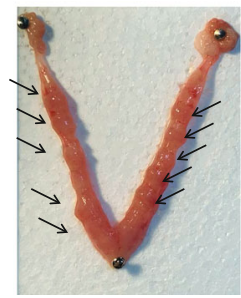

TJCYR-M+EDI

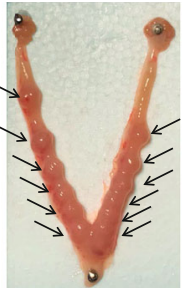

Prog+EDI

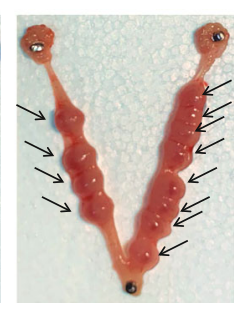

TJCYR-H+EDI (a)

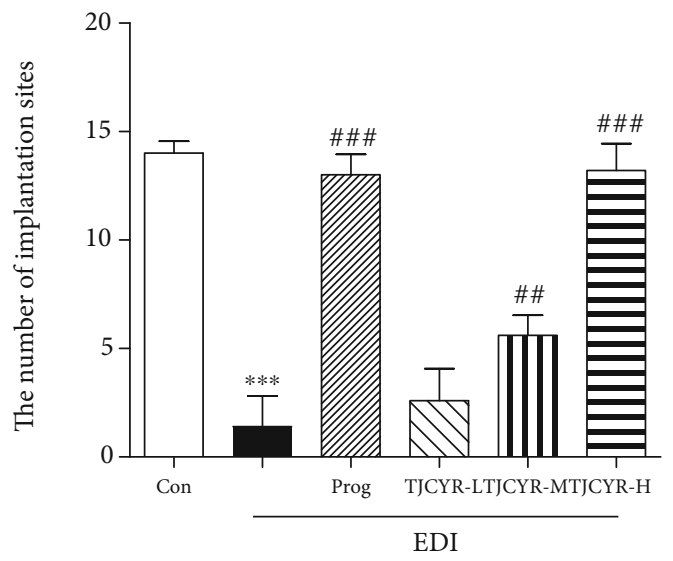

(b)

FiguRE 2: TJCYR increased the number of implantation sites. (a) A representative photograph showing the number of implantation sites (arrows) at Pd8; (b) quantification of implantation sites $(n=8)$. Results are expressed as mean \pm SEM. ${ }^{* * *} P<0.001$ versus control; ${ }^{\# \#} P<0.05$ and ${ }^{\# \# \#} P<0.01$ versus EID only. EID: embryo implantation dysfunction; Prog: progesterone; TJCYR: Tiaojing Cuyun Recipe; TJCYR-H: high-dose TJCYR; TJCYR-M: medium-dose TJCYR; TJCYR-L: low-dose TJCYR.

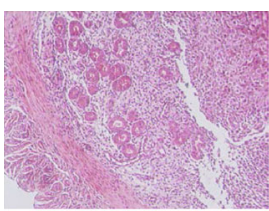

Con

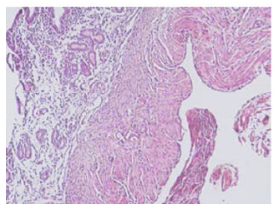

Prog+EID

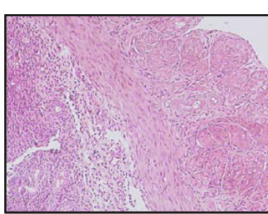

EID

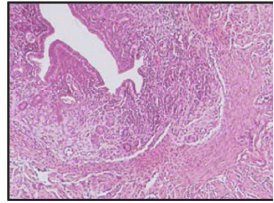

TJCYR+EID

(a)

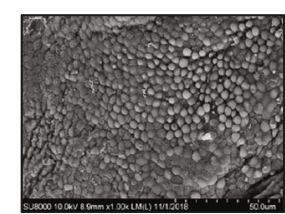

Con

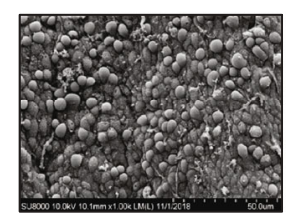

Prog+EID

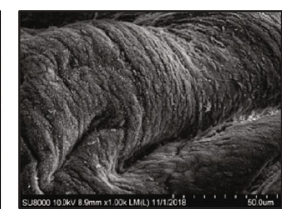

EID

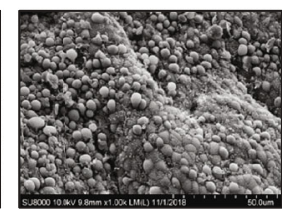

TJCYR+EID

(b)

Figure 3: Effect of TJCYR on EID-induced changes in endometrial morphology. (a) H\&E showing pathological changes in the endometrium $(\times 200, n=2)$; (b) SEM showing ultrastructure changes in pinopodes $(n=2)$.

related to endometrial receptivity, including Integrin $\alpha \mathrm{V}$, Integrin $\beta 3$, LIF, and OPN. As shown in Figure 5, immunofluorescence and Western blot analysis showed that the expression of Integrin $\alpha \mathrm{V}$, Integrin $\beta 3$, LIF, and OPN significantly decreased in the EID-only group. TJCYR was found to attenuate the EID-induced damage by increasing the expression of Integrin $\alpha \mathrm{V}$, Integrin $\beta 3$, LIF, and OPN. These results are consistent with the changes found in pinopodes as shown in Figure 3(b).

3.6. TJCYR Treatment Promotes Endometrial Angiogenesis. Angiogenesis is one of the important biological events which may be involved in implantation. Tomato Lectin is recognized as the most sensitive vessel marker, the fluorescence intensity of which may reflect vascular density. As shown in Figure 6(a), high immunofluorescence was detected using Tomato Lectin in the control and TJCYR treatment groups, while the immunofluorescence appeared weak in the EID only group. In contrast, microvascular density was dramatically enhanced in the TJCYR group. VEGF is an important mediator of angiogenesis with beneficial effects on endometrial receptivity and plays a key role in the embryonic development of mice. As shown in Figures 6(b) and 6(c), treatment with TJCYR clearly inhibited the decrease of VEGF in mice subjected to EID, which is consistent with the immunofluorescence analysis (Figure 6(a)) which showed increased vascular density.

3.7. TJCYR Improved Angiogenesis through the PI3K/Akt/ eNOS Signaling Pathway. The PI3K/Akt/eNOS pathway is downstream of VEGF, which participates in angiogenesis. Here, we found that the expressions of $\mathrm{p}$-Akt and p-eNOS were all reduced in the EID-only group compared to the control group, accompanied by a decline in NO. All of these 


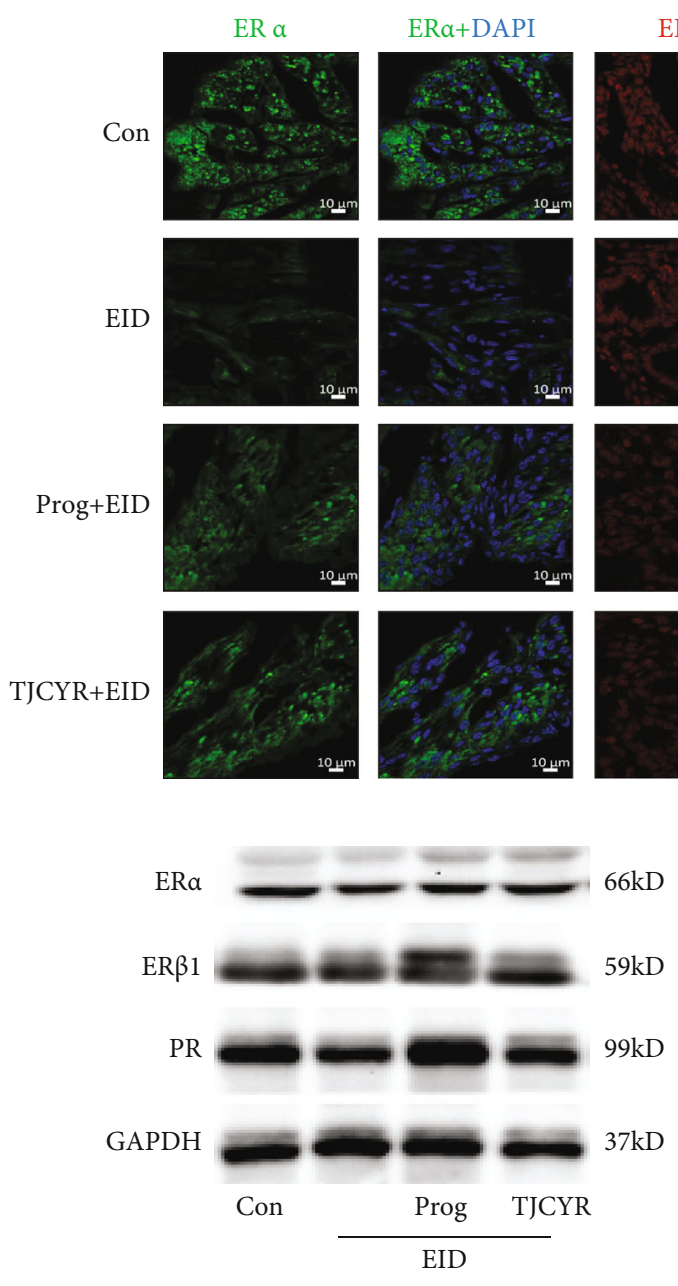

(b)

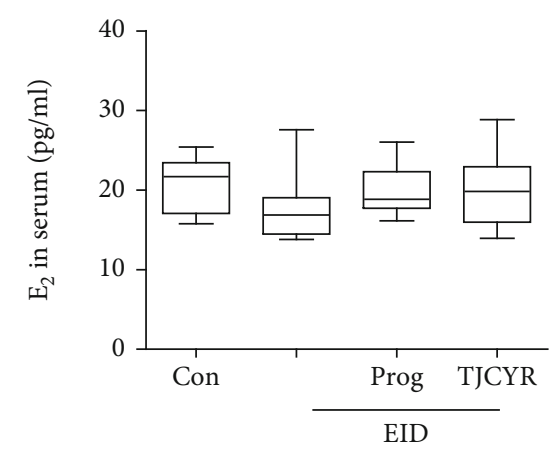

ER $\beta 1$
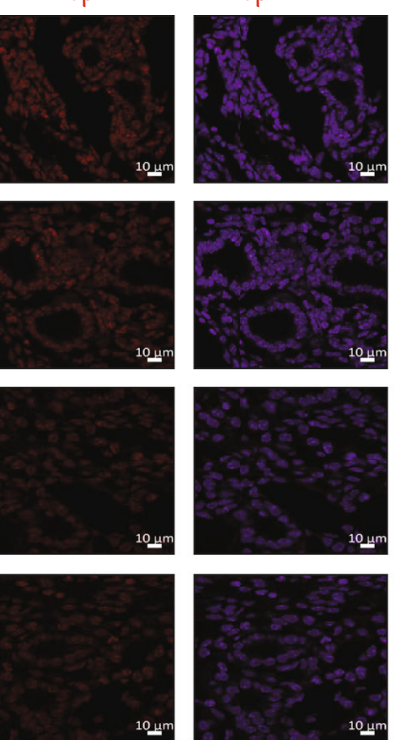

(a)
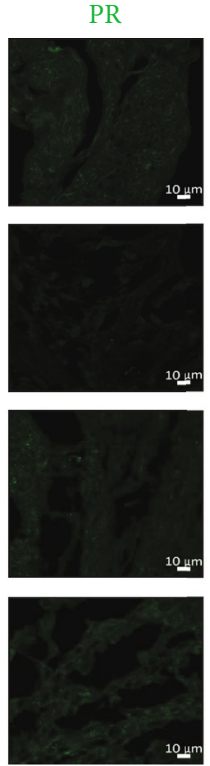

$10 \mathrm{~nm}$

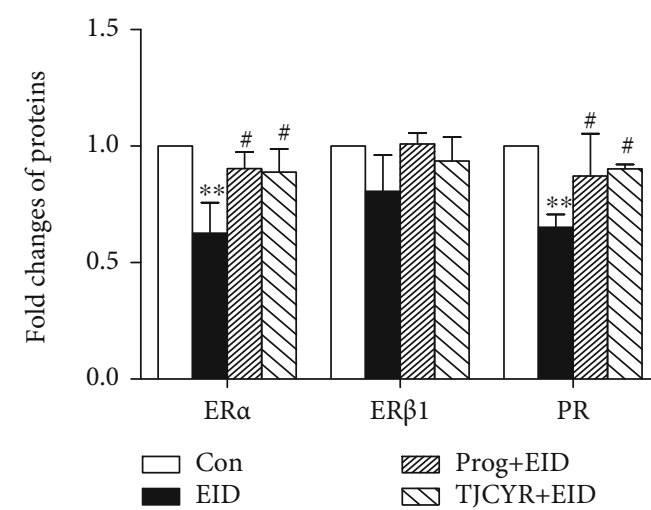

(c)

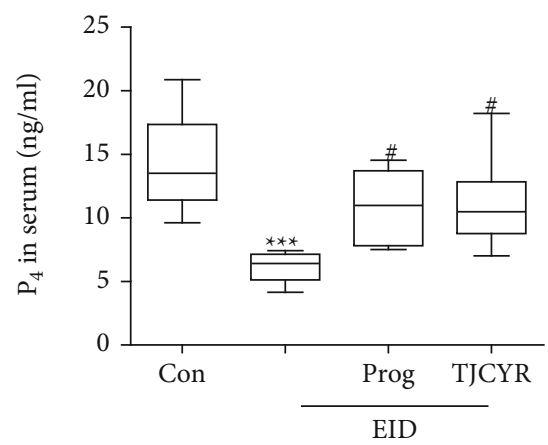

(d)

FIGURE 4: Effect of TJCYR on EID-induced changes in the hormones and receptors. (a) Representative confocal fluorescent images of the uterine sample with ER $\alpha$ (green), ER $\beta 1$ (red), and PR (green), respectively $(\times 20, n=2)$. (b) Protein levels of ER $\alpha$, ER $\beta 1$, and PR in uterine tissue were determined by Western blotting $(n=3)$; (c) quantification of protein levels. (d) The serum levels of $\mathrm{E}_{2}$ and $\mathrm{P}_{4}$ were tested by radioimmunoassay. Results are expressed as mean \pm SEM. ${ }^{* * *} P<0.001$ and ${ }^{* *} P<0.01$ versus control; ${ }^{\#} P<0.05$ versus EID only. EID: embryo implantation dysfunction; Prog: progesterone; TJCYR: Tiaojing Cuyun Recipe; $\mathrm{E}_{2}: 17 \beta$-estradiol; $\mathrm{P}_{4}$ : progesterone; ER $\alpha$ : estrogen receptor alpha; ER $\beta 1$ : estrogen receptor beta1; PR: progesterone receptor. 


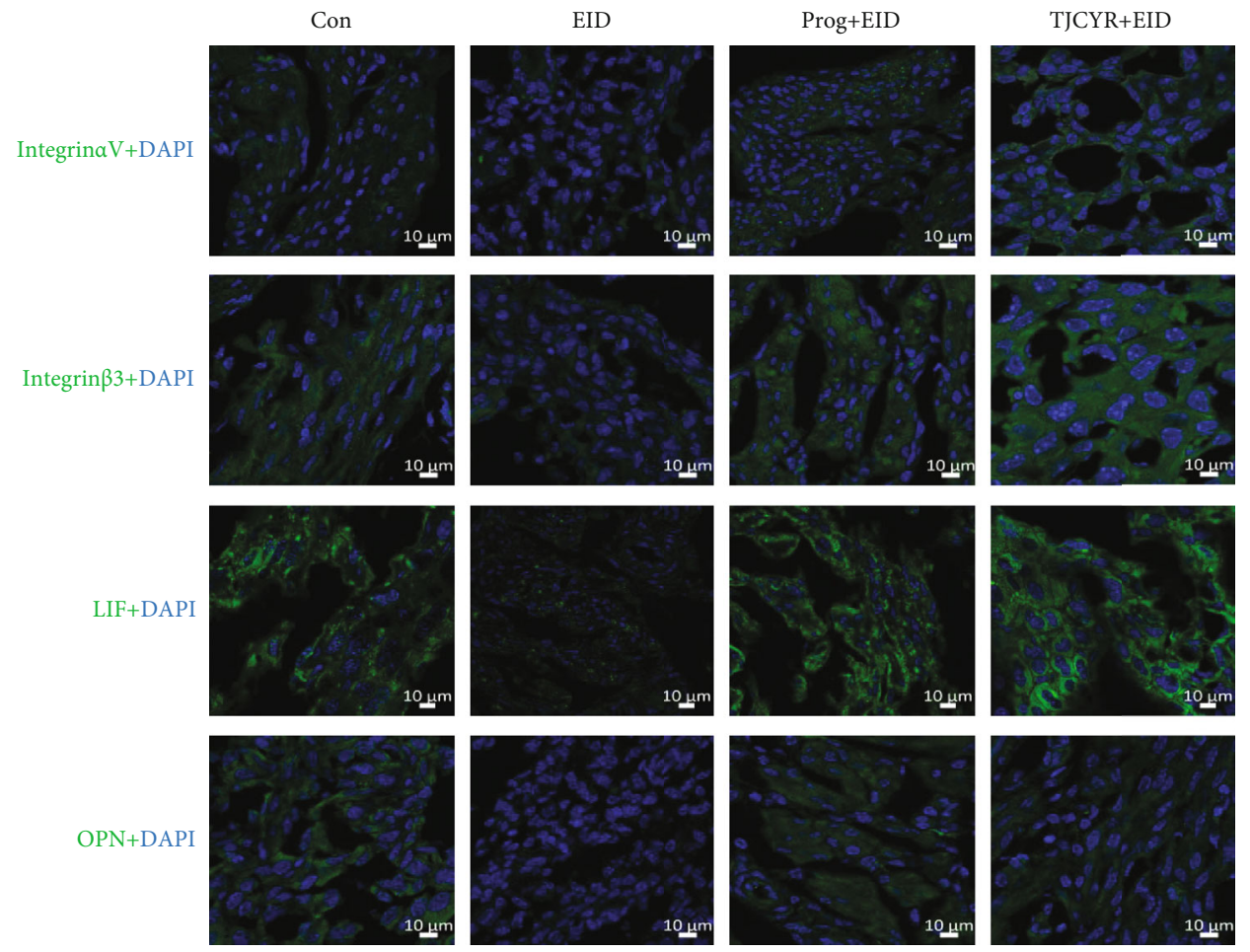

(a)

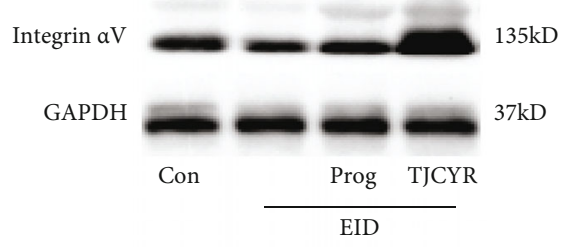

Integrin $\beta 3$

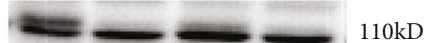

GAPDH

$37 \mathrm{kD}$

Con $\frac{\text { Prog TJCYR }}{\text { EID }}$

LIF

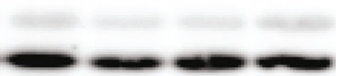

$22 \mathrm{kD}$

GAPDH

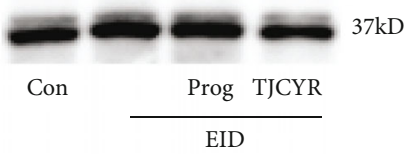

OPN

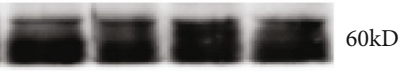

GAPDH

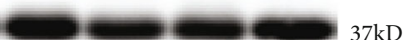

Con

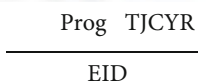

(b)

Figure 5: Continued. 

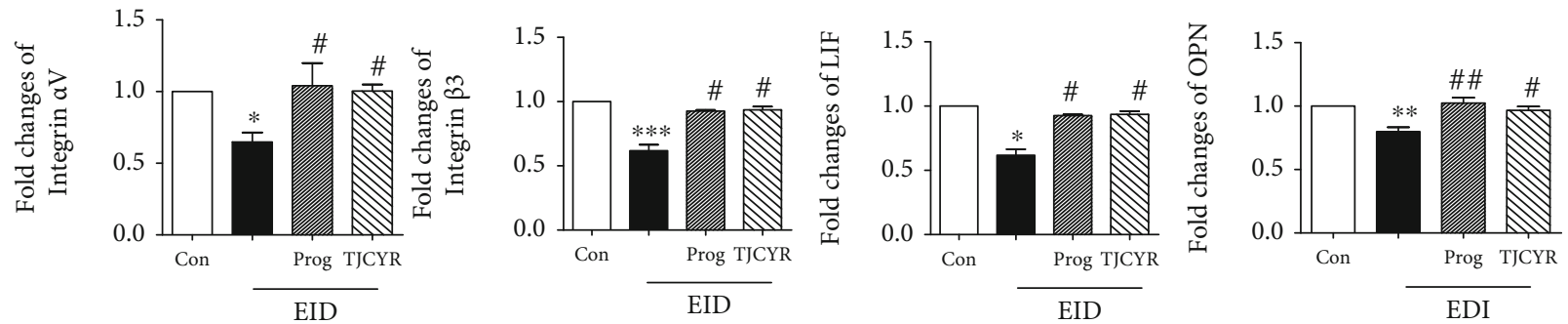

(c)

FiguRE 5: Effect of TJCYR on the biomarkers of endometrial receptivity. (a) Representative confocal fluorescent images of the uterine sample with Integrin $\alpha \mathrm{V}$, Integrin $\beta 3$, LIF, and OPN, respectively $(\times 20, n=2)$. (b) Protein levels of Integrin $\alpha \mathrm{V}$, Integrin $\beta 3$, LIF, and OPN in uterine tissue were determined by Western blotting $(n=3)$; (c) Quantification of protein levels. Results are expressed as mean \pm SEM. ${ }^{*} P$ $<0.05,{ }^{* *} P<0.01$, and ${ }^{* * *} P<0.001$ versus control; ${ }^{\#} P<0.05$ and ${ }^{\# \#} P<0.01$ versus EID only. EID: embryo implantation dysfunction; Prog: progesterone; TJCYR: Tiaojing Cuyun Recipe.

decreased expressions were restored by treatment with TJCYR (Figure 7). These data suggest that TJCYR stimulated the PI3K/Akt/eNOS signaling pathway, which was deactivated by EID.

\section{Discussion}

Successful embryo implantation is a highly orchestrated process involving blastocyst-uterine interactions. While blastocyst quality has been extensively studied, endometrial receptivity is equally important. Endometrial receptivity is defined as "that period of endometrial maturation during which the trophectoderm of the blastocyst can attach to the endometrial epithelial cells and subsequently proceed to invade the endometrial stroma and vasculature" [14]. Therefore, improving endometrial receptivity is key to raising the pregnancy rate. The pinopodes have been considered as the characteristic morphologic markers of endometrial receptivity and of the implantation window [13, 15]. The pinopodes are membrane protrusions on the apical surface of luminal epithelium on $\mathrm{Pd} 4$ in mice. They prevent cilia from sweeping the blastocyst away as well as promoting withdrawal of uterine fluid and closure of the uterine cavity $[4,16]$. In our study, the specimens were examined by SEM to detect pinopodes. The results revealed that TJCYR may enhance the number of fully developed pinopodes, accompanied by reduced distribution of cilia, which may provide nutrients for the embryo and enable its attachment to the uterine endometrium. The pinopodes play a key role in the initial stage of implantation by promoting attachment of the embryo.

The endometrium is the most direct target organ for estrogen and progesterone, among which E2 and P4 are the most important regulatory factors. The formation of pinopodes depends on the influence of estrogen and progesterone, the physiological effects of which depend on the mediation of estrogen receptors and progesterone receptors in endometrial glandular epithelial cells [17]. Therefore, estrogen, progesterone, and their receptors are important factors for the formation of good endometrial receptivity. Some studies $[18,19]$ have shown that the establishment of endometrial receptivity requires sufficient estrogen stimulation before ovulation and the supportive effect of progester- one on the endometrium after ovulation. In this study, we described the protective effects of TJCYR against EID, and we found that progesterone and its receptor were increased notably with TJCYR treatment.

Pregnancy is a versatile and dynamic process for the implanting embryo, requiring a series of rather complicated and synchronous morphological and biochemical changes. The appearance of pinopodes is consistent with the expression of several molecular markers of endometrial receptivity, including Integrin, leukemia inhibitory factor (LIF), and osteopontin (OPN). It is meaningful to analyze pinopodes and the molecular biomarkers together, hence our further studies exploring the effect of TJCYR on the expression of Integrin, LIF, and OPN. Integrins are cell surface receptors that are involved in cell-to-cell and extracellular matrix adhesion. Some Integrins increase during the implantation window. There are many isoforms of Integrins in mammals, but only three $(\alpha 1 \beta 1, \alpha 4 \beta 1$, and $\alpha \mathrm{V} \beta 3)$ have been found to have roles in implantation, $\alpha \mathrm{V} \beta 3$ playing the most conspicuous role [16]. Integrin $\alpha \mathrm{V} \beta 3$ is a potential receptor for blastocyst attachment and is localized on pinopodes [16]. Some studies have revealed that blockage of Integrin $\alpha \mathrm{V} \beta 3$ or lack of Integrin $\beta 3$ may be related to unexplained infertility [20]. LIF is a member of the interleukin- 6 family of cytokines, which plays a critical role in implantation. Pinopodes release secretory vesicles containing LIF in the uterine lumen to enable trophoblast invasion and affecting immune tolerance during implantation [16, 21]. Some studies [22] have shown that the deletion or mutation of LIF induced implantation failure. Other studies [23, 24] reported that endometrial LIF and receptor were higher around the time of implantation in fertile women compared with women with unexplained infertility. Other findings [25] have shown that LIF may maintain the proper development of the endometrium and implantation receptivity by regulating downstream target genes. OPN is one of the cofactors involved in cell adhesion and invasion during the implantation process, is an acidic member of the small integrin-binding ligand family of proteins [26], and has been shown to be maximally expressed in the epithelial layer in human, mouse, and rabbit uterine. OPN is therefore an important constituent of the uterus during pregnancy $[26,27]$. In this study, the EID group showed histopathological lesion characteristic of 


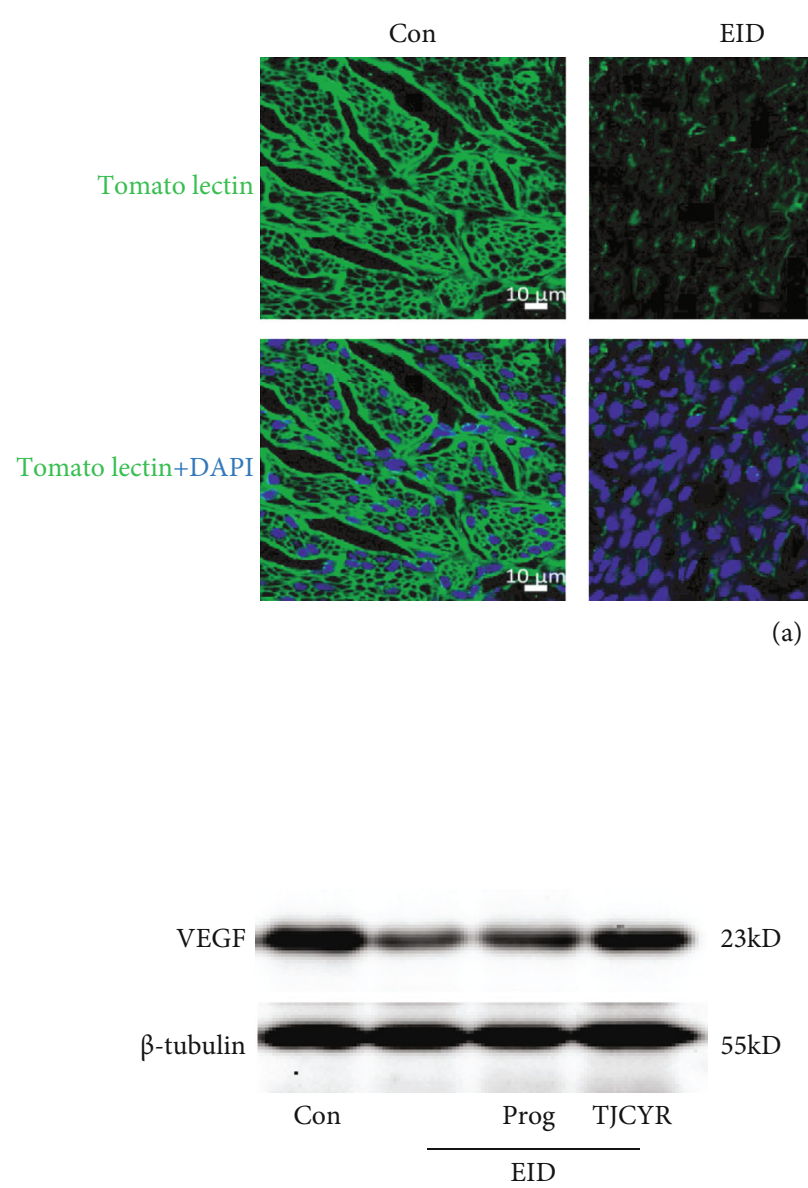

(b)
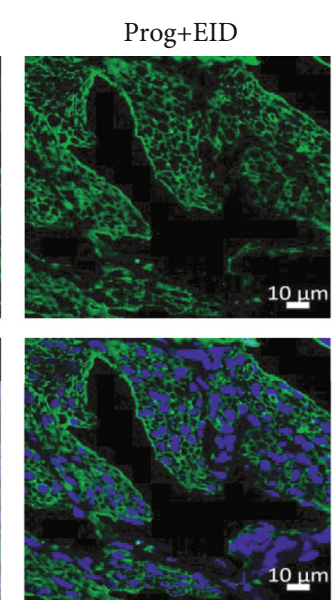

a)

Figure 6: Effect of TJCYR on angiogenesis and hypoxia. (a) Representative confocal fluorescent images of the uterine sample with Tomato Lectin (green) and DAPI (blue) $(\times 20, n=2)$; (b) protein levels of VEGF in uterine tissue were determined by Western blotting $(n=3)$; (c) quantification of protein levels. Results are expressed as mean $\pm \mathrm{SEM} ;{ }^{*} P<0.05$ and ${ }^{* *} P<0.01$ versus control; ${ }^{*} P<0.05$ versus EID only. EID: embryo implantation dysfunction; Prog: progesterone; TJCYR: Tiaojing Cuyun Recipe.

pinopodes, as well as low expression of Integrin $\alpha \mathrm{V}$, Integrin $\beta 3$, LIF, and OPN, while the expression of these biochemical markers was significantly increased in mice treated with TJCYR. These data strongly demonstrate the benefits of TJCYR in endometrial receptivity.

During the implantation window, a rich vascular network is necessary to supply nutrients and oxygen. As is well known, oxygen is abundant where blood supply is rich [28]. In clinical practice, blood flow scanning is the current and preferred approach for assessing endometrial function [29]. It is well established that proper endometrial vascular development and maintenance are crucial for successful pregnancy [30]. Insufficient angiogenesis may result in poor endometrial receptivity [31]. Based on Lectin immunostaining showing higher vascular density in TJCYR treatment, we next explored whether VEGF expression is increased with this treatment. VEGF has a predominant role in successful implantation and maintenance of pregnancy by increasing vascular permeability or forming vascular networks. It has been reported that VEGF knockout mice do not produce viable offspring [32]. The expression of VEGF increases significantly during implantation windows, which suggests that it promotes angiogenesis and the establishment of capillary networks, further improving endometrial receptivity and promoting embryo implantation [33]. Alternatively, deficiency of VEGF may induce the reduction of angiogenesis at the implantation site and lead to miscarriage [34]. VEGF inhibitors are used to achieve contraception [35]. In this study, we found a poor vascular network in the mice treated with EID only; that treatment with TJCYR significantly inhibited the decline in vessel density and expression of VEGF in the EID-only group and improved endometrial blood circulation. All the data showed that VEGF regulated angiogenesis and built endometrial microenvironment for embryo implantation.

It is well known that, as the downstream signaling pathway of VEGF, the activated PI3K/Akt pathway regulates cell proliferation, differentiation, apoptosis, cell cycle, protein synthesis, cell energy metabolism, and other functions [36, 37]. It had been demonstrated that the role of the PI3K/ Akt pathway may vary completely with conditions. In this study, we found that after the PI3K/Akt signaling pathway was activated by increased VEGF, the eNOS was sequentially activated. eNOS acts as a key regulator of angiogenesis by 


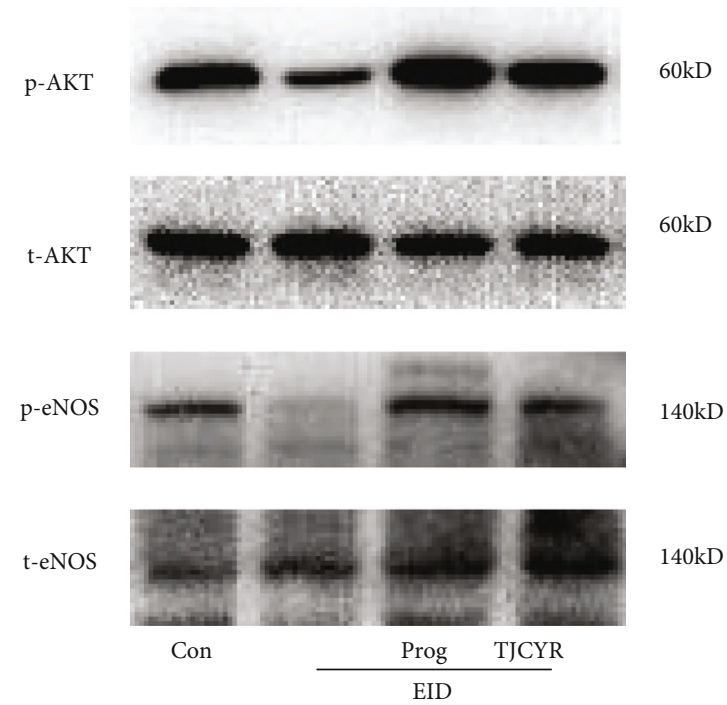

(a)
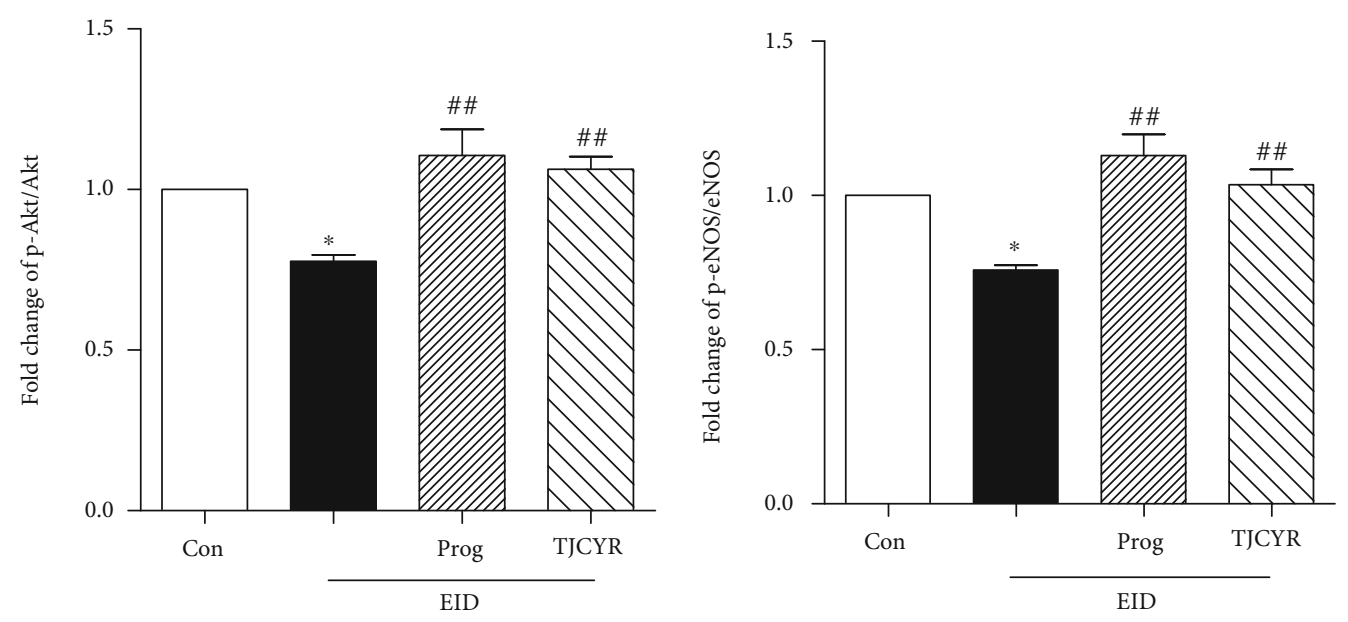

(b)

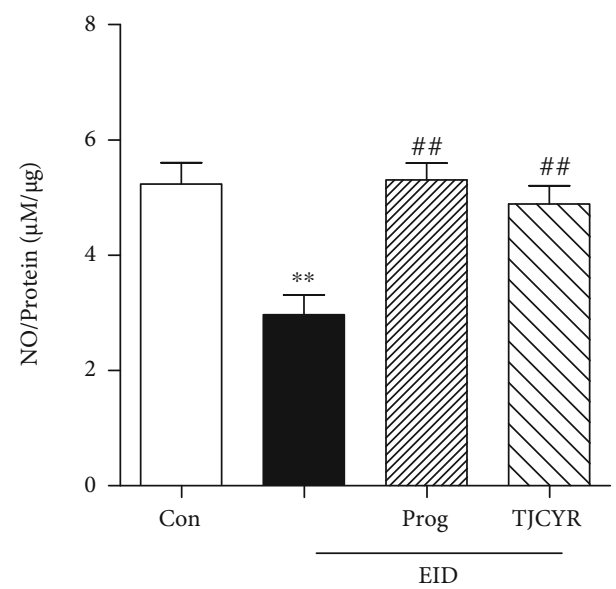

(c)

Figure 7: Effect of TJCYR on the PI3K/Akt/eNOS signaling pathway. (a) Protein levels of the PI3K/Akt/eNOS signaling pathway in uterine tissue were determined by Western blotting $(n=3)$; (b) quantification of protein levels. Results are expressed as mean \pm SEM; (c) NO production was shown by NO/protein $(n=10)$. Results are expressed as mean \pm SEM. ${ }^{*} P<0.05$ and ${ }^{* *} P<0.01$ versus control; ${ }^{\# \#} P<0.01$ versus EID only. EID: embryo implantation dysfunction; Prog: progesterone; TJCYR: Tiaojing Cuyun Recipe; NO: nitric oxide. 
mediating the speed of NO enzyme generation. NO is an important angiogenic substance produced by healthy endothelial cells to support vascular homeostasis and blood flow. During the reproductive processes, NO plays a critical role in maintaining blood vessel stability at the implantation site, further safeguarding embryo implantation and pregnancy maintenance. In the present study, we found that the expressions of p-Akt and p-eNOS were all reduced in the EID mice compared to the control group. Conversely, treatment with TJCYR markedly increased the expression of VEGF and subsequently increased the activation of the $\mathrm{PI} 3 \mathrm{~K} / \mathrm{Akt} / \mathrm{eNOS}$ pathway.

\section{Conclusion}

The results of the present study revealed that TJCYR can activate the PI3K/AKT/eNOS signaling pathway to improve endometrial microcirculation and blood flow, enhancing endometrial receptivity and embryo implantation, which were correlated with the characteristic changes in endometrial morphology and the upregulation of molecular markers for endometrial receptivity.

\section{Data Availability}

The data are available upon direct request to the corresponding author.

\section{Disclosure}

Part of the manuscript has previously been presented as a preprint in doi:10.21203/rs.3.rs-132200/v1 available via the following link: https://www.researchsquare.com/article/rs132200/v1

\section{Conflicts of Interest}

The authors declare that they have no conflict of interest regarding the publication of this paper.

\section{Authors' Contributions}

Li Dong conceived the study and designed the experiments. Hongli Huang, Lei Xia, Yanqiu Xia, Yunping Yan, Zhuojun Jiang, and Pei Zhao performed the experiments. Hongli Huang, Lei Xia, and Pei Zhao analyzed the data. Pei Zhao drafted the manuscript. Li Dong reviewed and revised the manuscript. Li Dong and Pei Zhao contributed equally to this paper. Hongli Huang and Lei Xia contributed equally to this work.

\section{Acknowledgments}

This study was funded by the Shanghai Science and Technology Committee project (No. 16401931900), the Natural Science Foundation of Shanghai (No. 16ZR1438000), the “Three-year" Development Project of Shanghai Shenkang Hospital Development Center (No. 16CR3040A), and the Budget Research Project of Shanghai Education Commission (No. 2019LK091).

\section{References}

[1] J. C. Harper, K. Aittomäki, P. Borry et al., "Recent developments in genetics and medically assisted reproduction: from research to clinical applications," Human Reproduction Open, vol. 2017, no. 3, 2017.

[2] E. R. Norwitz, D. J. Schust, and S. J. Fisher, "Implantation and the survival of early pregnancy," The New England Journal of Medicine, vol. 345, no. 19, pp. 1400-1408, 2001.

[3] L. A. Salamonsen, T. Edgell, L. J. F. Rombauts et al., "Proteomics of the human endometrium and uterine fluid: a pathway to biomarker discovery," Fertility and Sterility, vol. 99, no. 4, pp. 1086-1092, 2013.

[4] H. Matsumoto, "Molecular and cellular events during blastocyst implantation in the receptive uterus: clues from mouse models," The Journal of Reproduction and Development, vol. 63, no. 5, pp. 445-454, 2017.

[5] J. Cha, X. Sun, and S. K. Dey, "Mechanisms of implantation: strategies for successful pregnancy," Nature Medicine, vol. 18, no. 12, pp. 1754-1767, 2012.

[6] J. A. Miravet-Valenciano, A. Rincon-Bertolin, F. Vilella, and C. Simon, "Understanding and improving endometrial receptivity," Current Opinion in Obstetrics \& Gynecology, vol. 27, no. 3, pp. 187-192, 2015.

[7] X. Yu, C. Gao, C. Dai, F. Yang, and X. Deng, "Endometrial injury increases expression of hypoxia-inducible factor and angiogenesis in the endometrium of women with recurrent implantation failure," Reproductive BioMedicine Online, vol. 38, no. 5, pp. 761-767, 2019.

[8] A. Kim, H. Jung, W. J. Choi, S. N. Hong, and H. Y. Kim, "Detection of endometrial and subendometrial vasculature on the day of embryo transfer and prediction of pregnancy during fresh in vitro fertilization cycles," Taiwanese Journal of Obstetrics \& Gynecology, vol. 53, no. 3, pp. 360-365, 2014.

[9] Y. Lin and L. Dong, "Treatment of anovulatory infertility with shen deficiency syndrome by ZHU's Tiaojing Cuyun Recipe: a clinical evaluation," Chinese Journal of Integrated Traditional and Western Medicine, vol. 35, no. 10, pp. 1181-1185, 2015.

[10] H. L. Huang, L. Xia, Y. Q. Xia, Y. P. Yan, Z. J. Jiang, and L. Dong, "Effect of Tiaojing Zhuyun Recipe on ovulation induction in androgen-causing sterile rats," Journal of Shanghai University of Traditional Chinese Medicine, vol. 29, no. 2, pp. 61-66, 2015.

[11] J. Tao and L. Dong, "Effect of Bushen Huoxue formula on ACT-INH-FS system in patients with polycystic ovary syndrome of the kidney deficiency and blood stasis pattern," Shanghai Journal of Traditional Chinese Medicine, vol. 48, no. 7, pp. 58-61, 2014.

[12] N. Yu, W. Yan, Y. Wang, T. Yin, Y. Guo, and J. Yang, "Effect of Zhuyun recipe on endometrial pinopode expression in mice with embryonic implantation dysfunction and ovulation stimulation," Experimental and Therapeutic Medicine, vol. 9, no. 2, pp. 488-492, 2015.

[13] D. M. Huang, L. G. Nardo, G. Y. Huang, F. E. Lu, and Y. J. Liu, "Effect of a single dose of mifepristone on expression of pinopodes in endometrial surface of mice," Acta Pharmacologica Sinica, vol. 26, no. 2, pp. 212-219, 2005.

[14] B. A. Lessey and S. L. Young, "What exactly is endometrial receptivity?," Fertility and Sterility, vol. 111, no. 4, pp. 611$617,2019$.

[15] K. E. Quinn, B. C. Matson, M. Wetendorf, and K. M. Caron, "Pinopodes: recent advancements, current perspectives, and 
future directions," Molecular and Cellular Endocrinology, vol. 501, 2020.

[16] F. Z. Rarani, F. Borhani, and B. Rashidi, "Endometrial pinopode biomarkers: molecules and microRNAs," Journal of Cellular Physiology, vol. 233, no. 12, pp. 9145-9158, 2018.

[17] M. Wetendorf and F. J. DeMayo, “The progesterone receptor regulates implantation, decidualization, and glandular development via a complex paracrine signaling network," Molecular and Cellular Endocrinology, vol. 357, no. 1-2, pp. 108-118, 2012.

[18] C. Zijiang and Y. Junhao, "Latest research advances on factors that influence endometrial receptivity," Journal of Shandong University, vol. 59, no. 8, pp. 1-7, 2021.

[19] F. E. I. Gong, X. Li, S. Zhang et al., “A modified ultra-long pituitary downregulation protocol improved endometrial receptivity and clinical outcome for infertile patients with polycystic ovarian syndrome," Experimental and Therapeutic Medicine, vol. 10, no. 5, pp. 1865-1870, 2015.

[20] A. Germeyer, R. F. Savaris, J. Jauckus, and B. Lessey, "Endometrial beta3 integrin profile reflects endometrial receptivity defects in women with unexplained recurrent pregnancy loss," Reproductive Biology and Endocrinology, vol. 12, no. 1, pp. 5357, 2014.

[21] M. Kabir-Salmani, H. Nikzad, S. Shiokawa, Y. Akimoto, and M. Iwashita, "Secretory role for human uterodomes (pinopods): secretion of LIF," Molecular Human Reproduction, vol. 11, no. 8, pp. 553-559, 2005.

[22] H. Achache and A. Revel, "Endometrial receptivity markers, the journey to successful embryo implantation," Human Reproduction Update, vol. 12, no. 6, pp. 731-746, 2006.

[23] L. Aghajanova, "Leukemia inhibitory factor and human embryo implantation," Annals of the New York Academy of Sciences, vol. 1034, no. 1, pp. 176-183, 2004.

[24] E. Dimitriadis, C. Stoikos, M. Stafford-Bell et al., "Interleukin11, IL-11 receptoralpha and leukemia inhibitory factor are dysregulated in endometrium of infertile women with endometriosis during the implantation window," Journal of Reproductive Immunology, vol. 69, no. 1, pp. 53-64, 2006.

[25] J. G. Cheng, J. R. Chen, L. Hernandez, W. G. Alvord, and C. L. Stewart, "Dual control of LIF expression and LIF receptor function regulate Stat 3 activation at the onset of uterine receptivity and embryo implantation," Proceedings of the National Academy of Sciences of the United States of America, vol. 98, no. 15 , pp. $8680-8685,2001$.

[26] G. A. Johnson, R. C. Burghardt, F. W. Bazer, and T. E. Spencer, "Osteopontin: roles in implantation and placentation1," Biology of Reproduction, vol. 69, no. 5, pp. 1458-1471, 2003.

[27] Y. J. Kang, K. Forbes, J. Carver, and J. D. Aplin, "The role of the osteopontin-integrin $\alpha \mathrm{v} \beta 3$ interaction at implantation: functional analysis using three different in vitro models," Human Reproduction, vol. 29, no. 4, pp. 739-749, 2014.

[28] M. Y. Zhang, H. H. Chen, J. Tian et al., "Danggui Shaoyao San ameliorates renal fibrosis via regulation of hypoxia and autophagy," Evidence-Based Complementary and Alternative Medicine, vol. 2019, Article ID 2985270, 10 pages, 2019.

[29] L. Craciunas, I. Gallos, J. Chu et al., "Conventional and modern markers of endometrial receptivity: a systematic review and meta-analysis," Human Reproduction, vol. 25, no. 2, pp. 202-223, 2019.

[30] M. Zbucka, M. Koda, J. Tomaszewski, W. Przystupa, S. Sulkowski, and S. Wołczyński, "Angiogenesis in the female reproductive processes," Ginekologia Polska, vol. 75, no. 8, pp. 649-657, 2004.

[31] Q. Si and R. Liu, "Screening of angiogenesis inhibitors using a 3D vascular microfluidic chip to achieve contraception," Biochemical and Biophysical Research Communications, vol. 515, no. 1, pp. 92-98, 2019.

[32] P. Licht, V. Russu, S. Lehmeyer, T. Wissentheit, E. Siebzehnrübl, and L. Wildt, "Cycle dependency of intrauterine vascular endothelial growth factor levels is correlated with decidualization and corpus luteum function," Fertility and Sterility, vol. 80, no. 5, pp. 1228-1233, 2003.

[33] A. Ylikorkala, D. J. Rossi, N. Korsisaari et al., "Vascular abnormalities and deregulation of VEGF in Lkb1-deficient mice," Science, vol. 293, no. 5533, pp. 1323-1326, 2001.

[34] G. E. Lash, B. A. Innes, J. A. Drury, S. C. Robson, S. Quenby, and J. N. Bulmer, "Localization of angiogenic growth factors and their receptors in the human endometrium throughout the menstrual cycle and in recurrent miscarriage," Human Reproduction, vol. 27, no. 1, pp. 183-195, 2012.

[35] A. M. Sharkey, R. Catalano, A. Evans, D. S. Charnock-Jones, and S. K. Smith, "Novel antiangiogenic agents for use in contraception," Contraception, vol. 71, no. 4, pp. 263-271, 2005.

[36] J. S. Yu and W. Cui, "Proliferation, survival and metabolism: the role of PI3K/Akt/mTOR signalling in pluripotency and cell fate determination," Development, vol. 143, no. 17, pp. 30503060, 2016.

[37] P. Li, Y. Gan, Y. Xu et al., "The inflammatory cytokine TNF- $\alpha$ promotes the premature senescence of rat nucleus pulposus cells via the PI3K/Akt signaling pathway," Scientific Reports, vol. 7, no. 1, 2017. 